\title{
Entropy of Soft Sets
}

\author{
K.Y. Qin \\ College of Mathematics, Southwest Jiao tong University \\ Chengdu, Sichuan, China
}

\begin{abstract}
This paper is devoted to the discussion of uncertainty measures of soft sets. We make an analysis of the existing works on soft set entropy and show their limitations. We propose a new axiomatic definition of soft set entropy. Furthermore, some distance based entropies for soft sets are presented.
\end{abstract}

Keywords-soft set; fuzzy sets; the distance between soft sets; soft set entropy

\section{INTRODUCTION}

To solve complicated problems in economics, engineering, environmental science and social science, methods in classical mathematics are not always successful because of various types of uncertainties presented in these problems. While probability theory, fuzzy set theory [1], rough set theory [2], and other mathematical tools are wellknown and often useful approaches to describing uncertainty, each of these theories has its inherent difficulties as pointed out in [3, 4]. Consequently, Molodtsov [3] proposed a completely new approach for modeling vagueness and uncertainty in 1999. This approach called soft set theory is free from the difficulties affecting existing methods.

Accordingly, works on soft set theory are progressing rapidly. Maji et al. [5] defined several algebraic operations on soft sets and conducted a theoretical study on the theory of soft sets. Based on [5], Ali et al. [6] introduced some new operations on soft sets and improved the notion of complement of soft set. They proved that certain De Morgan's laws with respect to these new operations hold in soft set theory. Qin et al. [7] introduced the notion of soft equality and established lattice structures and soft quotient algebras of soft sets. Maji et al. [8] initiated the study on hybrid structures involving soft sets and fuzzy sets. They proposed the notion of fuzzy soft set as a fuzzy generalization of classical soft sets and some basic properties were discussed. Afterwards, many researchers have worked on this concept. Various kinds of extended fuzzy soft sets have been proposed.

The study of uncertainty measures is an important topic for the theories to deal with uncertainty. Majumdar and Samanta $[9,10]$ initiated the study of uncertainty measures of soft sets and fuzzy soft sets. Some similarity measures between soft sets and fuzzy soft sets were presented. Kharal [11] introduced some set operations based distance and similarity measures for soft sets. Wang and $\mathrm{Qu}$ [12] introduced axiomatic definitions of entropy, similarity measure and distance measure for vague soft sets, and some formulas have also been put forward to calculate them.

\author{
J.L. Yang \\ College of Fundamental Education \\ Sichuan Normal University \\ Chengdu, Sichuan, China
}

Based on fuzzy implication operators and fuzzy equivalences, Qin et al. [13] proposed a new category of inclusion measures and similarity measures for fuzzy soft sets. Liu et al. [14] proposed some entropy for fuzzy soft sets. We notice that the entropies presented in [10, 12-14] are based on the entropies for fuzzy sets. If the fuzzy soft sets (resp. vague soft sets) degenerate to soft sets, the entropies will be zero (take minimum value). Thus the uncertainty degree of soft set cannot be described. Majumdar and Samanta [15] pointed out that the soft sets deals with the uncertainty arising from the parameterized classification of objects of a universe. They proposed the axiomatic definition of softness measure for soft sets. This measure is isotone with respect to a specific preference relation between soft set. Some softness measures were presented. This paper is devoted to a further study of the uncertainty measures of soft sets. Some limitations in the existing papers were pointed out. We propose a new axiomatic definition of soft set entropy. Additionally, some new soft set entropies were presented.

\section{PRELIMINARIES}

This section presents a review of some fundamental notions and conclusions of soft sets. We refer to $[3,6,7]$ for details.

The concept of soft sets is proposed by Molodtsov [3] in 1999. Let $U$ be the universe set and $E$ the set of all possible parameters under consideration with respect to $U$. Usually, parameters are attributes, characteristics, or properties of objects in $U .(U, E)$ is called a soft space. Molodtsov defined the notion of a soft set in the following way:

Definition 1. [3] A pair $(F, A)$ is called a soft set over $U$, where $A \subseteq E$ and $F$ is a mapping given by $F: A \rightarrow P(U)$, and $P(U)$ is the power set of $U$.

In other words, a soft set over $U$ is a parameterized family of subsets of $U$. For ${ }^{e \in A}, F(e)$ may be considered as the set of $e$-approximate elements of the soft set $(F, A)$.

Maji ea al. [5] and Ali et al. [6] proposed some operations on soft sets. Based on these operations, Qin et al. [7] established the lattice structure of soft sets.

Definition 2. [6] Let $(F, A)$ and $(G, B)$ be two soft sets over a common universe $U$. 
(1) The extended union of $(F, A)$ and $(G, B)$, denoted by $(F, A) \cup_{e}(G, B)$, is the soft set $(H, C)$, where $C=A \cup B$, and $H$ is given by:

$$
H(a)=\left\{\begin{array}{lrr}
F(a), & \text { if } & a \in A-B, \\
G(a), & \text { if } & a \in B-A, \\
F(a) \cup G(a), & \text { if } & a \in A \cap B .
\end{array}\right.
$$

(2) The extended intersection of $(F, A)$ and $(G, B)$, denoted by $(F, A) \cap_{e}(G, B)$, is the soft set $(H, C)$, where $C=A \cup B$, and $H$ is given by:

$$
H(a)=\left\{\begin{array}{lll}
F(a), & \text { if } & a \in A-B, \\
G(a), & \text { if } & a \in B-A, \\
F(a) \cap G(a), \text { if } & a \in A \cap B .
\end{array}\right.
$$

(3) The restricted union of $(F, A)$ and $(G, B)$, denoted by $(F, A) \bigcup_{r}(G, B)$, is the soft set $(H, C)$, where $C=A \cap B$, and $H(a)=F(a) \cup G(a)$ for every $a \in C$.

(4) The restricted intersection of $(F, A)$ and $(G, B)$, denoted by $(F, A) \cap_{r}(G, B)$, is the soft set $(H, C)$, where $C=A \cap B$, and $H(a)=F(a) \cap G(a)$ for every $a \in C$.

Definition 3. [6] (1) $(F, A)$ is called a null soft set (with respect to the parameter set $A$ ), denoted by $\varnothing_{A}$, if $F(e)=\varnothing$ for all $e \in A$.(2) $(G, A)$ is called a absolute soft set (with respect to the parameter set $A$ ), denoted by $U_{A}$, if $F(e)=U$ for all $e \in A$.

Definition 4. The complement of a soft set $(F, A)$ is denoted by $(F, A)^{c}$ and is defined by $(F, A)^{c}=\left(F^{c}, A\right)$, where $F^{c}: A \rightarrow P(U)$ is a mapping given by $F^{c}(e)=U-F(e)$ for all $e \in A$.

Clearly, $\left((F, A)^{c}\right)^{c}=(F, A)$.

Definition 5. [15] A soft set $(F, A)$ over $U$ is said to be a deterministic soft set if $\bigcup_{e \in A} F(e)=U$ and $F\left(e_{1}\right) \cap F\left(e_{2}\right)=\varnothing$ whenever $e_{1}, e_{2} \in A$ and $e_{1} \neq e_{2}$.

By this definition, $(F, A)$ is a deterministic soft set if $\{F(e) ; e \in A\}-\{\varnothing\}$ is a partition of the universe $U$.We denote by $S(U, E)$ the set of all soft sets over the universe $U$ and the parameter set $E$, that is

$$
S(U, E)=\{(F, A) ; A \subseteq E, F: A \rightarrow P(U)\} .
$$

Theorem 1. [7] (1) $\left(S(U, E), \bigcup_{e}, \bigcap_{r}\right)$ is a bounded distributive lattice, $U_{E}$ and $\varnothing_{\varnothing}$ are the upper bound and lower bound respectively. (2) Let $\leq$ be the ordering relation in lattice $\left(S(U, E), \bigcup_{e}, \cap_{r}\right)$ and $(F, A),(G, B) \in S(U, E)$. $(F, A) \leq(G, B)$ if and only if $A \subseteq B$ and $F(e) \subseteq G(e)$ for each $e \in A$.

\section{ANALYSIS OF SOFT SET ENTROPY PRESENTED IN [15]}

Majumdar [15] pointed out that the associated uncertainty of a soft set arises from the parameterized classification of objects in the universe. Let $(F, A)$ be a soft set over the universe $U$. An object $x \in U$ can be classified by a parameter $e \in A$ if $x \in F(e)$. The entropy, i.e. the associated uncertainty, of a soft set should be maximum if either the objects cannot be classified at all w.r.t the parameters or every object of the universe can be classified by every parameter. Again the entropy of a soft set is minimum if each object can be classified just by one parameter. Thus, the entropies of null soft set and absolute soft set are maximum, whereas the entropy of deterministic soft set is minimum. Furthermore, for a superset of a soft set the uncertainty ultimately increases in comparison with its subset, as new objects being introduced in the set which shares same parameters with other objects. Based on this observation, Majumdar [15] proposed the notion of equivalent soft sets and the axiomatic definition of soft set entropy.

Definition 6. [15] Let $(F, A)$ and $(G, A)$ be two soft sets over $U .(F, A)$ is said to be equivalent to $(G, A)$ if there exists a bijective mapping $\sigma: A \rightarrow A$ such that $\sigma\left(F_{x}\right)=G_{x}$ for every $x \in U$, where $F_{x}=\{e \in A ; x \in F(e)\} \quad$ and $G_{x}=\{e \in A ; x \in G(e)\}$.

Let $C(F, A)$ denote the collection of all soft sets which are equivalent to $(F, A)$.

Definition 7. [15] Let $(U, E)$ be a soft space. A mapping $f: S(U, E) \rightarrow[0,1]$ is said to be soft set entropy or softness measure if $f$ satisfies the following properties:

$(\mathrm{S} 1)^{f}\left(\varnothing_{A}\right)=f\left(U_{A}\right)=1$.

(S2) $f(F, A)=0$ if $(F, A)$ is a deterministic soft set.

$(\mathrm{S} 3)^{f}(F, A) \leq f(G, A)$ if $(F, A) \neq \varnothing_{A}$ and $(F, A) \subseteq(G, A)$.

(S4) $f\left(F^{*}, A\right)=f(F, A)$ for $\left(F^{*}, A\right) \in C(F, A)$.

Theorem 2. [15] The function $f_{M}: S(U, E) \rightarrow[0,1]$ is a soft set entropy, where $f_{M}\left(\varnothing_{A}\right)=f_{M}\left(U_{A}\right)=1$, and $f_{M}(F, A)=1-\frac{|U|}{\sum_{x \in U}|\{e \in A ; x \in F(e)\}|}$ otherwise.

Let $U=\left\{x_{1}, x_{2}, \cdots, x_{n}\right\}, A=\left\{e_{1}, e_{2}, \cdots, e_{m}\right\}$ and $(F, A)$ be a soft set over $U$. We recall that $(F, A)$ can be expressed as a binary $n \times m$ table [1]. If $x_{i} \in F\left(e_{j}\right)$ then $h_{i j}=1$, otherwise $h_{i j}=0$, where $h_{i j}$ are the entries in the table. For each $x_{i} \in U,\left|\left\{e \in A ; x_{i} \in F(e)\right\}\right|$ is the number of 1's appearing in ith row and hence $\sum_{x_{i} \in U}\left|\left\{e \in A ; x_{i} \in F(e)\right\}\right|$ is the number of 
1 's appearing in the whole table. On the other hand, $\left|F\left(e_{j}\right)\right|$ is the number of 1's appearing in jth column. Thus $\sum_{e_{j \in A}}\left|F\left(e_{j}\right)\right|$ is also the number of 1's appearing in the table. Accordingly, we have the following corollary.

Corollary 1. If $(F, A) \neq \varnothing$ and $(F, A) \neq U_{A}$, then $f_{M}(F, A)=1-\frac{|U|}{\sum_{e \in A}|F(e)|}$.

Definition 8. [15] Let $(U, E)$ be a soft space and $(F, A)$ a soft set over the universe $U$.

(1) The nearest soft set of $(F, A)$ is a soft set $\left(F_{\text {near }}, A\right)$ which is a deterministic over soft set over $R_{T}$ and is obtained from $(F, A)$ by eliminating the least number of elements, where $R_{T}=\bigcup_{e \in A} F(e)$ is the range of F.

(2) The farthest soft set of $(F, A)$ is a soft set $\left(F_{\text {far }}, A\right)$ which is obtained from $(F, A)$ by including additional elements in $(F, A)$ such that for every $e \in A, F_{f a r}(e)=R_{T}$.

Let $(U, E)$ be a soft space, $U=\left\{x_{1}, x_{2}, \cdots, x_{n}\right\}, A \subseteq E$ and $A=\left\{e_{1}, e_{2}, \cdots, e_{m}\right\}$.

Definition 9. For two soft sets $(F, A)$ and $(G, A)$, the mean Hamming distance $d((F, A),(G, A))$ is defined as [9]:

$$
\begin{aligned}
& d((F, A),(G, A))=\frac{1}{m} \sum_{i=1}^{m} \sum_{j=1}^{n}\left|F\left(e_{i}\right)\left(x_{j}\right)-G\left(e_{i}\right)\left(x_{j}\right)\right| \\
& \text { Where } F\left(e_{i}\right)\left(x_{j}\right)=1 \text { if } x_{j} \in F\left(e_{i}\right) \text {, and } F\left(e_{i}\right)\left(x_{j}\right)=0 \text { if } \\
& x_{j} \notin F\left(e_{i}\right) .
\end{aligned}
$$

Let $d_{1}$ and $d_{2}$ be the distances between $(F, A)$ and $\left(F_{\text {near }}, A\right),(F, A)$ and $\left(F_{\text {far }}, A\right)$ respectively. It is noted that the nearest soft set of a soft set is not unique. But the distances between a soft set and its nearest soft sets are the same.

Definition 10. [15] (1) A non null non absolute soft set $(F, A)$ is said to be a soft set of TYPE-I, if $d_{1} \leq d_{2}$. (2) A non null non absolute soft set $(F, A)$ is said to be a soft set of TYPE-II, if $d_{1}>d_{2}$.

The distance based softness measure or soft entropy of $(F, A)$ is defined as [15]:

$$
E(F, A)= \begin{cases}d_{1} / d_{2}, & \text { if }(F, A) \neq \varnothing_{A},(F, A) \neq U_{A}, d_{1} \leq d_{2}, \\ d_{2} / d_{2}, & \text { if }(F, A) \neq \varnothing_{A},(F, A) \neq U_{A}, d_{2} \leq d_{1}, \\ 1, & \text { if }(F, A)=\varnothing_{A} \vee(F, A)=U_{A} .\end{cases}
$$

Theorem 3. (Theorem 3.13 [15]) The softness measures defined in (3) satisfy all the properties of Definition 10 for TYPE-I soft sets.
Example 1. Let $U=\left\{x_{i} ; 1 \leq i \leq 7\right\}, A=\left\{e_{1}, e_{2}, e_{3}\right\}$. We consider the soft sets $(F, A)$ and $(G, A)$, where $F\left(e_{1}\right)=\left\{x_{1}, x_{3}\right\} \quad, \quad F\left(e_{2}\right)=\left\{x_{2}, x_{3}, x_{4}\right\} \quad F\left(e_{3}\right)=\left\{x_{1}, x_{4}\right\}$; $G\left(e_{1}\right)=\left\{x_{1}, x_{3}, x_{5}\right\}, G\left(e_{2}\right)=\left\{x_{2}, x_{3}, x_{4}, x_{6}\right\}, G\left(e_{3}\right)=\left\{x_{1}, x_{4}, x_{7}\right\}$.

(1) For soft set $(F, A),\left(F_{\text {near }}, A\right)$ is defined as: $F_{\text {near }}\left(e_{1}\right)=\left\{x_{1}\right\}, F_{\text {near }}\left(e_{2}\right)=\left\{x_{2}, x_{3}\right\}, F_{\text {near }}\left(e_{3}\right)=\left\{x_{4}\right\}$. Again $\left(F_{f a r}, A\right)$ is defined as $F_{f a r}\left(e_{i}\right)=\left\{x_{1}, x_{2}, x_{3}, x_{4}\right\}$ for each $1 \leq i \leq 3$. It follows that $d_{1}=1 / 3(1+1+1)=1$, $d_{2}=1 / 3(2+1+2)=5 / 3$. Thus $(F, A)$ is TYPE-I soft set and $E(F, A)=\frac{d_{1}}{d_{2}}=3 / 5$.

(2) For soft set $(G, A),\left(G_{\text {near }}, A\right)$ is defined as:

$$
G_{\text {near }}\left(e_{1}\right)=\left\{x_{1}, x_{5}\right\} \quad, \quad G_{\text {near }}\left(e_{2}\right)=\left\{x_{2}, x_{3}, x_{6}\right\}
$$

$G_{\text {near }}\left(e_{3}\right)=\left\{x_{4}, x_{7}\right\}$. Again $\left(G_{\text {far }}, A\right)$ is defined as $G_{\text {far }}\left(e_{i}\right)=\left\{x_{1}, x_{2}, x_{3}, x_{4}, x_{5}, x_{6}, x_{7}\right\}$ for each $1 \leq i \leq 3$. It follows that $d_{1}=1 / 3(1+1+1)=1, \quad d_{2}=1 / 3(4+3+4)=11 / 3$. Thus $(G, A)$ is TYPE-I soft set and $E(G, A)=d_{1} / d_{2}=3 / 11$.

In this example we have $(F, A) \subseteq(G, A)$ and $E(F, A)>E(G, A)$. Thus the monotonicity (S3) does not hold. It shows that Theorem 3.13 presented in [15] is not correct.

\section{A New CATEGory of SOFT SET ENTROPy}

The entropy measure quantifies the degree of uncertainty. In 1965, Zadeh introduced the fuzzy entropy for the first time [16]. Luca and Termini [17] introduced the axiom construction of entropy of fuzzy sets by using the similar concept of Shannon's probability entropy in which they gave an axiom definition of entropy of fuzzy sets. The entropies of fuzzy sets have been extensively studied. One general approach to entropy of fuzzy sets is based the distance between this fuzzy set and its complement. The following theorem shows that this method is not suitable for soft sets.

Theorem 4. Let $(U, E)$ be a soft space and $(F, A)$ a soft set over $U$. Then $d\left((F, A)\left(F^{c}, A\right)\right)=|U|$.

Proof. By the definition, we have $F^{c}(e)=U-F(e)$ for each $e \in A$. It follows that $\left|F(e)(x)-F^{c}(e)(x)\right|=1$ for every $x \in U$ hence

$$
\begin{aligned}
& \sum_{x \in U}\left|F(e)(x)-F^{c}(e)(x)\right|=|U| \text {. Consequently } \\
& d\left((F, A)\left(F^{c}, A\right)\right)=\frac{1}{A} \sum_{e \in A} \sum_{x \in U}\left|F(e)(x)-F^{c}(e)(x)\right|=\frac{1}{|A|} \sum_{e \in A}|U|=|U|
\end{aligned}
$$

By this theorem, the distance between a soft set and its 
complement is always the cardinality $|U|$ of the universe. Thus we cannot use it to characterize uncertainty of soft set.

Theorem 5. Let $(U, E)$ be a soft space.

(1) $G_{1}\left(\varnothing_{A}\right)=G_{1}\left(U_{A}\right)=|U|$.

$G_{1}(F, A)=|| U\left|-\frac{2}{A} \sum_{e \in A}\right| F(e)||$.

(3) $0 \leq G_{1}(F, A) \leq|U|$.

Proof. (1) is trivial. (2) Let $(F, A)$ be a soft set over $U$. We have

$$
\begin{gathered}
d\left((F, A), \varnothing_{A}\right)=1 /|A| \sum_{e \in A} \sum_{x \in U} F(e)(x)=1 /|A| \sum_{e \in A}|F(e)|, \\
d\left((F, A), U_{A}\right)=1 /|A| \sum_{e \in A} \sum_{x \in U}|F(e)(x)-1|=1 /|A| \sum_{e \in A}|U-F(e)| .
\end{gathered}
$$

It follows that:

$$
\begin{aligned}
& G_{1}(F, A)=\left|d\left((F, A), \varnothing_{A}\right)-d\left((F, A), U_{A}\right)\right|=|1 /| A\left|\sum_{e \in A}\right| F(e)|-1 /| A\left|\sum_{e \in A}\right| U-F(e)|| \\
& =1 / A\left|\sum_{e \in A}\right| F(e)\left|-\sum_{e \in A}(U|-| F(e) \mid)\right|=1 /\left.|A|\right|^{2} \sum_{e \in A}|F(e)|-|A||U| \\
& =|U|-2 /|A| \sum_{e \in A}|F(e)| .
\end{aligned}
$$

(3) For each $e \in A$, we have $|F(e)| \leq|U|$, and hence $0 \leq \sum_{e \in A}|F(e)| \leq|A||U|$. Thus $0 \leq 2 /|A| \sum_{e \in A}|F(e)| \leq 2|U|$, and $-|U| \leq|U|-2 /|A| \sum_{e \in A}|F(e)| \leq|U| \quad, \quad$ and consequently $0 \leq G_{1}(F, A) \leq|U|$ as required.

Based on the idea of fuzzy set entropy, for a soft set $(F, A)$, we may think that the more $(F, A)$ and $\left(F^{c}, A\right)$ are close to each other in some sense, the bigger its uncertainty. We propose the following new definition of soft set entropy, called TYPE-II soft set entropy.

Definition 11. Let $(U, E)$ be a soft space. A mapping $E: S(U, E) \rightarrow[0,1]$ is said to be TYPE-II soft set entropy if $E$ satisfies the following properties:

(1) $E\left(\varnothing_{A}\right)=E\left(U_{A}\right)=0$

(2) $E(F, A)=E\left(F^{c}, A\right)$ for any $(F, A) \in S(U, E)$.

(3) For any $(F, A),(G, A) \in S(U, E)$, if $\left|d\left((F, A), \varnothing_{A}\right)-d\left(\left(F^{c}, A\right), \varnothing_{A}\right)\right| \leq\left|d\left((G, A), \varnothing_{A}\right)-d\left(\left(G^{c}, A\right), \varnothing_{A}\right)\right|$ , then $E(G, A) \leq E(F, A)$.

Theorem 6. Let $(U, E)$ be a soft space. ${ }^{E_{1}}$ is a TYPE-II soft set entropy, where $E_{1}(F, A)=1-1 /|U|^{G_{1}(F, A)}$, for each $(F, A) \in S(U, E)$.

Proof. By $0 \leq G_{1}(F, A) \leq|U|$, it is clear that $0 \leq E_{1}(F, A) \leq 1$
(1) By $G_{1}\left(\varnothing_{A}\right)=|U|$, it follows that $E_{1}\left(\varnothing_{A}\right)=1-1 / U G_{1}\left(\varnothing_{A}\right)=0$. Similarly, we have $E_{1}\left(U_{A}\right)=0$. (2) is trivial. (3) Let $(F, A),(G, A) \in S(U, E)$, and

$$
\left|d\left((F, A), \varnothing_{A}\right)-d\left(\left(F^{c}, A\right), \varnothing_{A}\right)\right| \leq\left|d\left((G, A), \varnothing_{A}\right)-d\left(\left(G^{c}, A\right), \varnothing_{A}\right)\right|
$$

. Thus we have $G_{1}(F, A) \leq G_{1}(G, A)$ and consequently

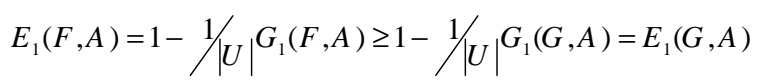

Theorem 7. Let $(U, E)$ be a soft space. ${ }^{E_{2}}$ is a TYPE-II soft set entropy, where

$$
E_{2}(F, A)=\frac{d\left((F, A), \varnothing_{A}\right) \wedge d\left(\left(F^{c}, A\right), \varnothing_{A}\right)}{d\left((F, A), \varnothing_{A}\right) \vee d\left(\left(F^{c}, A\right), \varnothing_{A}\right)}
$$$$
(F, A) \in S(U, E)
$$

The proof is similar to that of Theorem 6 .

\section{CONCLUDING REMARKS}

Soft sets and fuzzy soft sets are mathematical tools for dealing with uncertainties. This paper is devoted to a further study of the uncertainty measures of soft sets. We proposed a new axiomatic definition of soft set entropy. Some formulas have also been put forward to calculate soft set entropy.

In further research, soft set entropy based on different distance measures and similarity measures is an important and interesting issue to be addressed.

\section{ACKNOWLEDGEMENTS}

This work has been supported by the National Natural Science Foundation of China (Grant No. 61473239, 61175044), The Program of Education Office of Sichuan province (Grant No.11ZB068) and The Fundamental Research Funds for the Central Universities of China (Grant No.2682014ZT28).

\section{REFERENCES}

[1] L.A.Zadeh, Fuzzy sets, Information and Control, 8, 338-353, 1965.

[2] Z.Pawlak, Rough sets, International Journal of Computer and Information Science, 11, 341-356, 1982.

[3] D. Molodtsov, Soft set theory-First results, Computers and Mathematics with Applications, 37, 19-31, 1999.

[4] D. Molodtsov, The theory of soft sets, URSS Publishers, Moscow (in Russian), 2004.

[5] P.K.Maji, R.Biswas, A.R.Roy, Soft set theory, Computers and Mathematics with Applications, 45, 555-562, 2003.

[6] M.I.Ali, F.Feng, X.Liu, W.K.Min, M.Shabir, On some new operations in soft set theory, Computers and Mathematics with Applications, 57, 1547-1553, 2009.

[7] K.Y.Qin, Z.Y.Hong, On soft equality, Journal of Computational and Applied Mathematics, 234, 1347-1355, 2010.

[8] P.K.Maji, R.Biswas, A.R.Roy, Fuzzy soft sets, The Journal of Fuzzy Mathematics, 9, 589-602, 2001.

[9] P.Majumdar, S.K.Samanta, Similarity measure of soft sets, New Mathematics and Natural Computation, 4(1), 1-12, 2008. 
[10] P.Majumdar, S.K.Samanta, On similarity measures of fuzzy soft sets, International Journal of Advance in Soft Computing and its Application, 3(2) 1-8, 2011.

[11] Kharal, Distance and similarity measures for soft sets, New Mathematics and Natural Computation, 6(3), 321-334, 2010.

[12] Wang, A.Qu, Entropy, similarity measure and distance measure of vague soft sets and their relations, Information Sciences, 244, 92-106, 2013.

[13] K.Y.Qin, Z.Y.Hong, Z.C.Liu, Some uncertainty measures of fuzzy soft sets, in: Proceedings of 2013 Asia-Pacific Computational Intelligence and Information Technology, D.Zhong and A.Hu Eds. Shanghai: DEStech Publications, 245-251, 2013.

[14] Z.C.Liu, K.Y.Qin, Z.Pei, Similarity measure and entropy of fuzzy soft sets, The ScientificWorld Journal, Volume 2014, Article ID 161607 , 10 pages, http://dx.doi.org/10.1155/2014/161607.

[15] P.Majumdar, S.K.Samanta, Softness of a soft sets: Soft set entropy, Annals of Fuzzy Mathematics and Informatics, 6(1), 59-68, 2013.

[16] L.A.Zadeh, Fuzzy sets and systems, in: Proceedings of the Symposium on Systems Theory, Polytechnic Institute of Brooklyn, NY, pp. 29-37, 1965.

[17] A.D.Luca, S.Termini, A definition of a nonprobability entropy in the setting of fuzzy sets theory, Information and Control, 20, 301-312, 1972. 